\title{
Các yếu tố ảnh hưởng đến sự tin tưởng và ý định tiếp tục sử dụng dịch vụ giao thực phẩm trên ứng dụng di động của người tiêu dùng
}

\section{The factors affect consumer's trust and continuous usage intention of food delivery mobile apps}

\author{
Hoàng Thị Phương Thảo ${ }^{*}$, Lâm Quí Long ${ }^{1}$ \\ ${ }^{1}$ Trường Đại học Mở Thành phố Hồ Chí Minh, Việt Nam \\ *Tác giả liên hệ, Email: hthiphuongthao@yahoo.com
}

\begin{tabular}{|c|c|}
\hline THÔNG TIN & TÓM TĂT \\
\hline $\begin{array}{l}\text { DOI:10.46223/HCMCOUJS. } \\
\text { econ.vi.16.2.931.2021 }\end{array}$ & $\begin{array}{l}\text { Nghiên cứu này nhằm ứng dụng mô hình chấp nhận và sử } \\
\text { dụng công nghệ mở rộng (UTAUT2) để phản ánh các yếu tố ảnh } \\
\text { hưởng đến ý định tiếp tục sử dụng dịch vụ giao thực phẩm trên } \\
\text { Ứng Dụng Di Động (ƯDDĐ) thông qua biến trung gian là sự tin } \\
\text { tưởng của Người Tiêu Dùng (NTD). Kết quả xử lý dữ liệu bằng } \\
\text { phần mềm SPSS và AMOS trên } 378 \text { phiếu khảo sát hợp lệ, đã chỉ }\end{array}$ \\
\hline Ngày nhận: 27/08/2020 & ra được có 08 yếu tố có tác động đến ý định tiếp tục sử dụng ứng \\
\hline Ngày nhận lại: 28/08/2020 & $\begin{array}{l}\text { dụng dạt hang thực pham gom co: (1) ky vọng hiẹu qua, (2) ky } \\
\text { vọng nỗ lực, (3) ảnh hưởng xã hội, (4) điều kiện thuận lợi, (5) }\end{array}$ \\
\hline Duyệt đăng: 10/09/2020 & $\begin{array}{l}\text { động lực thụ hưởng, (6) giá trị giá cả, (7) chất lượng thông tin và } \\
\text { (8) sự tin tưởng. Trong đó, } 03 \text { yếu tố ảnh hưởng lớn nhất đến ý } \\
\text { định tiếp tục sử dụng dịch vụ giao hàng thực phẩm là giá trị giá } \\
\text { cả, ảnh hướng xã hội và sự tin tưởng. Đồng thời kêt quả cũng cho } \\
\text { thấy có } 06 \text { yếu tố cũng tác động tích cực đến sự tin tưởng của } \\
\text { người tiêu dùng đối với ứng dụng gồm có: (1) kỳ vọng hiệu quả, } \\
\text { (2) kỳ vọng nồ lực, (3) ảnh hường xã hội, (4) điều kiện thuận lợi, } \\
\text { (5) giá trị giá cả và (6) chất lượng thông tin. }\end{array}$ \\
\hline
\end{tabular}

Tù khóa:

mô hình chấp nhận và sử dụng công nghệ (UTAUT2); ứng dụng di động; giao hàng thực phẩm; sự tin tưởng; ý định tiếp tục sử dụng

Keywords:

unified theory of acceptance and use of technology 2 (UTAUT2); mobile apps;

\section{ABSTRACT}

This study aims to apply the Unified Theory of Acceptance and Use of Technology 2 (UTAUT2) model to reflect the main factors that affect continuous usage intention of food delivery mobile apps through intervining variable of consumer's trust. Using SPSS and AMOS software on 378 answer sheets, data processing results show that 08 factors are impacting on continuous intention to use the food ordering application, including: (1) Performance Expectancy, (2) Effort Expectancy, (3) Social Influence, (4) Facilitating Conditions, (5) Hedonic Motivation, (6) Price Value, (7) Information Quality and (8) Trust. In particular, the three most important factors that influence the intention to continue using food delivery services are Price Value, Social Influence, and Trust. At the same time, the results also show that there are 06 factors that positively impact on consumer's trust of the application, including: (1) Performance Expectancy, (2) Effort Expectancy, (3) Social food delivery; consumer's trust; Influence, (4) Facilitating Conditions, (5) Price Value and (6) continuous intention Information Quality. 


\section{1. Đặt vấn đề}

Dịch vụ giao thực phẩm trên ứng dụng di động tại Việt Nam đang phát triển nhưng vẫn còn nhỏ bé so với thị trường trong khu vực và trên thế giới. Theo báo cáo của Statista (2020) thì quy mô thị trường tại Việt Nam chiếm tỉ lệ rất bé so với các nước trong khu vực Châu Á Thái Bình Dương và chỉ chiếm khoảng $0.6 \%$ so với thị trường giao đồ ăn trực tuyến đạt 45.6 tỷ đô trên thế giới. Theo báo cáo của E-Conomy SEA (2019), tại khu vực Đông Nam Á có tốc độ tăng trưởng hàng năm trong lĩnh vực giao thực phẩm trực tuyến từ năm 2015 đến 2019 lên tới $91 \%$ và ước đạt giá trị lên tới 20 tỉ đô năm 2025. Cũng theo báo cáo trên, trong vòng 04 năm từ năm 2016 đến năm 2019 thì thị trường giao thực phẩm trực tuyến tại Indonesia đã tăng trưởng 13 lần, Philippines tăng trưởng 09 lần, Thái Lan tăng trưởng 08 lần, trong khi tại Việt Nam thì chỉ tăng trưởng 02 lần.

Các yếu tố nhanh chóng, tiện lợi và số lượng thực phẩm trên ứng dụng đa dạng, dịch vụ giao thực phẩm đã giúp tiết kiệm thời gian, đáp ứng các sở thích ẩm thực của nhiều đối tượng NTD khác nhau, đặc biệt là người dân tại các thành phố lớn. Cùng với sự phổ biến và dễ dàng sử dụng của điện thoại thông minh, chỉ vài thao tác lựa chọn trên ứng dụng, NTD có thể đặt và nhận các món ăn ngay tại nhà mà không cần di chuyển tới các cửa hàng, quán ăn. Hơn nữa, trong thời kỳ dịch bệnh Covid 19 đang hoành hành, việc dùng U’DDĐ để đặt hàng thức ăn mang lại tiện dụng rất lớn cho NTD và hoàn toàn phù hợp trong hoàn cảnh giãn cách xã hội. Ngoài ra, việc dễ dàng và thuận tiện trong việc chọn lựa nhiều hình thức thanh toán khác nhau như tiền mặt, ví điện tử cũng giúp tạo nên sự thuận tiện trong việc sử dụng các ứng dụng này. Với những lợi ích có được từ dịch vụ giao hàng thực phẩm đã kích thích nhu cầu sử dụng dịch vụ này ngày càng tăng cao. Tuy nhiên, để duy trì khách hàng tiếp tục sử dụng dịch vụ là điều rất quan trọng nhằm tạo được sự khác biệt, tăng tính cạnh tranh và duy trì sự phát triển trong tương lai của doanh nghiệp.

Những nghiên cứu về ý định tiếp tục sử dụng dịch vụ trên nền tảng công nghệ xác định vị trí thu hút sự quan tâm đặc biệt của các nhà nghiên cứu gần đây. Các nghiên cứu trước đã chỉ ra khá nhiều các yếu tố khác nhau ảnh hưởng đến ý định tiếp tục sử dụng dịch vụ giao hàng thực phẩm trên UDDĐ. Dựa trên lý thuyết chấp nhận và sử dụng công nghệ mở rộng: các yếu tố hiệu suất mong đợi, ảnh hưởng xã hội và thói quen (Lee, Sung, \& Jeon, 2019), động lực thụ hưởng (Alalwan, 2019) ảnh hưởng lớn đến ý định tiếp tục sử dụng dịch vụ. El-Masri và Tarhini (2017) đã chỉ ra rằng sự tin tưởng cũng ảnh hưởng đến ý định tiếp tục sử dụng các dịch vụ và công nghệ mới. Các nghiên cứu còn cho thấy trong bối cảnh thị trường khác nhau, sản phẩm/dịch vụ khác nhau thì các yếu tố ảnh hưởng và mức độ ảnh hưởng cũng khác nhau khi sử dụng mô hình UTAUT2, do đó dịch vụ giao hàng thực phẩm trên UDDĐ là một bối cảnh cụ thể tại Việt Nam để đi sâu nghiên cứu về chủ đề đề này.

Mục tiêu của nghiên cứu này là xác định các yếu tố tác động đến sự tin tưởng và ý định tiếp tục sử dụng dịch vụ giao hàng thực phẩm trên UDDĐ tại thị trường Việt Nam. Dựa trên kết quả nghiên cứu, các hàm ý quản trị sẽ được đề xuất để để nâng cao chất lượng dịch vụ cũng như tăng tính cạnh tranh và thu hút NTD sử dụng thử và tiếp tục sử dụng dịch vụ mới mẻ này.

\section{Cơ sở lý thuyết và giả thuyết nghiên cứu}

\subsection{Dịch vụ giao thục phẩm trên UDD円}

Theo Alalwan (2019) thì dịch vụ giao thực phẩm trên U’DDĐ được định nghĩa là U’DDĐ mà người dùng điện thoại thông minh tải xuống và sử dụng. Bằng cách sử dụng các ứng dụng này, khách hàng có thể dễ dàng và hiệu quả hơn trong việc truy cập và đặt món ăn từ một loạt 
các quán ăn, nhà hàng có vị trí thuận tiện cho người dùng với thông tin toàn diện như thực đơn, giá, khuyến mãi, phương thức thanh toán, thời gian giao hàng dự kiến. Kèm theo thông tin này, khách hàng có thể thấy tiến trình đặt hàng thông qua tất cả các giai đoạn như thời gian giao hàng, người vận chuyển, lịch trình di chuyển. Các mặt hàng được giao có thể bao gồm các món khai vị, món chính, đồ uống, món tráng miệng, các thực phẩm đã qua chế biến có thể sử dụng tức thì.

\subsection{Mô hình lý thuyết chấp nhận và sử dụng công nghệ mở rộng UTAUT2}

Mô hình UTAUT2 do Venkatesh, Thong, và Xu (2012) phát triển từ mô hình chấp nhận công nghệ mở rộng - UTAUT1 (Venkatesh, Morris, Davis, \& Davis, 2003). Mục tiêu của mô hình UTAUT2 nhằm dự đoán hành vi chấp nhận và sử dụng công nghệ của một tổ chức hay cá nhân. Với việc bổ sung thêm ba yếu tố là động lực thụ hưởng, giá trị giá cả và thói quen so với mô hình cũ UTAUT1. Mô hình UTAUT2 có thể nói là đã khắc phục được sự không toàn diện của mô hình TRA (Ajzen \& Fishbein, 1975), TAM (Davis, 1989), TPB (Ajzen, 1991) và UTAUT1 trước đây và cũng đã được nhiều nhà nghiên cứu vận dụng trong các mô hình chấp nhận công nghệ mới của mình. Dựa trên mô hình UTAUT2, các nhà nghiên cứu có thể vận dụng theo mô hình gốc hoặc bổ sung thêm một số biến mới đề phù hợp với đặc điểm văn hóa và mức độ phát triển công nghệ của mỗi nước.

\subsection{Sụ tin tưởng và ý định tiếp tục sủ̉ dụng}

Ý định tiếp tục sử dụng là xu hướng tương lai của một khách hàng ở lại với đơn vị cung cấp sản phẩm hoặc dịch vụ (Ranaweera \& Prabhu, 2003). Việc duy trì khách hàng sử dụng tiếp tục sản phẩm hoặc dịch vụ là một chiến lược kinh doanh quan trọng đối với bất kỳ công ty nào. Các doanh nghiệp luôn cố gắng giảm thiểu tình trạng khách hàng từ bỏ sản phẩm của mình vì việc duy trì khách hàng ảnh hưởng trực tiếp đến lợi nhuận của doanh nghiệp. Theo nghiên cứu của Fleming và Asplund (2007), khi khách hàng gắn bó với sản phẩm/dịch vụ đối với doanh nghiệp sẽ tạo ra doanh thu cao hơn 1.7 lần so với khách hàng bình thường. Rosenberg và Czepiel (1984) chỉ ra rằng chi phí để tạo một khách hàng mới cao khoảng "06 lần” so với việc duy trì một khách hàng hiện tại. Do vậy, các doanh nghiệp ngày càng tập trung và nỗ lực để đảm bảo khách hàng tin tưởng, hài lòng và tiếp tục sử dụng sản phẩm/dịch vụ thay vì chỉ tập trung vào việc thu hút các khách hàng mới.

Trong kinh doanh, sự tin tưởng là nhận thức của NTD về sự tin cậy của thương hiệu, sản phẩm hoặc dịch vụ (Flavian, Guinaliu, \& Gurrea, 2006). Sự tin tưởng là sự sẵn sàng của một bên có nhiều rủi ro mong đợi rằng một bên khác sẽ thực hiện một hành động cụ thể giống với các yêu cầu, cam kết đã đưa ra mà không cần hoặc ít giám sát (Chai \& Kim, 2010). Theo Kustiwi và Isnalita (2018) cho rằng sự tin tưởng lĩnh vực kinh doanh là niềm tin giữa các bên liên quan đều hoàn thành tất cả các trách nhiệm của mình để việc giao dịch đạt được kết quả mong đợi và mang lại sự hài lòng cho tất cả. Sự tin tưởng trong thương mại điện tử là mức độ tin cậy cho thấy ý định và hành động của một NTD trong quá trình giao dịch (Wijoseno \& Ariyanti, 2017). Nghiên cứu của El-Masri và Tarhini (2017), Escobar-Rodríguez và Carvajal-Trujillo (2014) đã chứng tỏ rằng sự tin tưởng là yếu tố dự báo mạnh nhất về ý định mua hàng trực tuyến. Niềm tin càng lớn của NTD vào các trang web thương mại điện tử thì họ càng có ý định mua hàng và tiếp tục mua trong tương lai. Nghiên cứu của Indrawati và Putri (2018), Wijoseno và Ariyanti (2017) đã kết luận rằng sự tin tưởng vào việc thanh toán trên ỦDDĐ ảnh hưởng mạnh đến ý định tiếp tục sử dụng lại ứng dụng này trong tương lai. Dựa trên lược khảo lý thuyết, giả thuyết $\mathrm{H} 1$ được đề xuất như sau: 
H1: Sự tin tưởng ảnh huởng cùng chiều đến đến ý định tiếp tục sư dụng dịch vụ giao nhận thực phẩm trên ứng dụng di động của người tiêu dùng

\subsection{Kỳ vọng hiệu quả, sụ tin tưởng và ý định tiếp tục sủ̉ dụng}

Kỳ vọng hiệu quả được định nghĩa là mức độ mà một cá nhân tin rằng việc sử dụng hệ thống sẽ giúp họ có thể đạt được lợi ích trong hiệu suất công việc (Venkatesh et al., 2003). Biến kỳ vọng hiệu quả bắt nguồn từ nhận thức hữu dụng từ lý thuyết chấp nhận công nghệ TAM (Davis, 1989). Khái niệm kỳ vọng hiệu quả được chỉ ra đó là kỳ vọng về sự hữu dụng, tiết kiệm thời gian và tăng năng suất công việc của khách hàng. Kang và Namkung (2018) đã nhấn mạnh rằng NTD nhận thấy mức độ hiệu suất, hiệu quả ảnh hưởng tích cực đến niềm tin của khách hàng khi sử dụng dịch vụ giao hàng thực phẩm trực tuyến. Đối với ứng dụng dịch vụ giao thực phẩm, yếu tố kỳ vọng hiệu quả đã được kiểm tra là có tác động đến ý định tiếp tục sử dụng ứng dụng này (Alalwan, 2019; Lee et al., 2019). Từ cơ sở lý thuyết trên giả thuyết H2 và H3 được đề xuất như sau:

H2: Kỳ vọng hiệu quả ảnh hương cùng chiều đến sụ tin tưởng ưng dụng đặt hàng thực phẩm trên di động của người tiêu dùng

H3: Kỳ vọng hiệu quả ảnh hương cùng chiều đến ý định tiếp tục sủ dụng dịch vu giao nhận thực phẩm trên ưng dụng di động của người tiêu dùng

\subsection{Kỳ vọng nô̂ lục, sụ̂ tin tưởng và ý định tiếp tục sủ dụng}

Kỳ vọng nỗ lực được định nghĩa là mức độ dễ dàng liên quan đến việc sử dụng hệ thống. Theo Venkatesh và cộng sự (2003) thì yếu tố này bắt nguồn từ nhận thức dễ sử dụng. Davis (1989) chỉ ra rằng khi một cá nhân nhận thức có thể dễ dàng sử dụng một ứng dụng thì khả năng chấp nhận chúng càng cao. Khi một hệ thống không phức tạp, dễ sử dụng và dễ thao tác thì người dùng sẽ nhận thấy nhà cung cấp dịch vụ mang lại cho họ sự tiện lợi và từ đó làm tăng độ tin tưởng vào hệ thống đó (Gefen, Karahanna, \& Straub, 2003). Zhou (2012) cho rằng sự dễ sử dụng là một thuộc tính của việc xây dựng lòng tin trong các hệ thống di động. Khi NTD cảm thấy rằng một công nghệ quá phức tạp để kiểm soát thì lúc đó sẽ khó hình thành lòng tin do nhận thức về rủi ro tăng lên. Úng dụng đặt hàng thực phẩm trên di động yêu cầu khách hàng hoàn thành tất cả các quy trình đặt hàng thực phẩm mà không cần bất kỳ sự hỗ trợ từ nhân viên nhà hàng hay đơn vị cung cấp ứng dụng. Nghiên cứu của Alalwan (2019), Lee và cộng sự (2019) đã đánh giá việc dễ dàng sử dụng ứng dụng có tác động đáng kể đến ý định tiếp tục sử dụng ứng dụng đặt hàng thực phẩm trên di động. Nghiên cứu của Kustiwi và Isnalita (2018) khẳng định khả năng sử dụng ứng dụng dễ dàng sẽ dẫn đến tăng niềm tin của NTD và cải thiện doanh số bán hàng. Từ cơ sở lý thuyết trên giả thuyết $\mathrm{H} 4$ và $\mathrm{H} 5$ được đề xuất như sau:

H4: Kỳ vọng nỗ lục ảnh hương cùng chiều đến sụ tin tưởng ứng dụng đặt hàng thục phẩm trên di động của người tiêu dùng

H5: Kỳ vọng nô̂ lục ảnh hwởng cùng chiều đến ý định tiếp tục sủ dụng dịch vụ giao nhận thực phẩm trên ưng dụng di động của người tiêu dùng

\subsection{Anh hưởng xã hội, sụ tin tưởng và ý định tiếp tục sử dụng}

Ảnh hưởng xã hội được định nghĩa là mức độ mà một cá nhân cho rằng những người khác tin rằng họ nên sử dụng hệ thống mới (Venkatesh et al., 2003). Với những lợi ích về Internet mang lại, một trong số đó là dữ liệu và thông tin khổng lồ. Do vậy, việc sàng lọc thông tin chính xác và tin cậy để ra quyết định mua hàng bị ảnh hưởng bởi nhiều yếu tố, trong đó NTD sẽ tin tưởng hơn các sản phẩm và dịch vụ được giới thiệu bởi bạn bè và người thân của họ. Hơn nữa, những người nổi tiếng trong cộng đồng cũng có tầm ảnh hưởng lớn đến hành vi mua sắm 
của NTD. Nghiên cứu của Singh, Alryalat, Alzubi, và Sarma (2017) đã cho thấy yếu tố ảnh hưởng xã hội tác động đến về sự tin tưởng của NTD khi có ý định mua hàng trực tuyến. Trong nghiên cứu của Verkijika (2018), ông đã đánh giá rằng ảnh hưởng xã hội có vai trò đáng kể trong việc dự đoán ý định sử dụng ứng dụng thương mại trên di động của khách hàng. Nghiên cứu của Lee và cộng sự (2019) chỉ ra rằng ảnh hưởng xã hội là một trong các yếu tố ảnh hưởng tích cực đến ý định tiếp tục sử dụng ứng dụng giao hàng thực phẩm tại Hàn Quốc. Từ cơ sở lý thuyết trên giả thuyết $\mathrm{H6}$ và $\mathrm{H} 7$ được đề xuất như sau:

H6: Ảnh hưởng xã hội ảnh hưởng cùng chiều đến sụ tin tưởng úng dụng đặt hàng thưc phẩm trên di động của người tiêu dùng

H7: Anh hương xã hội ảnh hương cùng chiều đến ý định tiếp tục sủ dụng dịch vu giao nhận thực phẩm trên ứng dụng di động của người tiêu dùng

\section{7. Điều kiện thuận lọi, sụ tin tưởng và ý định tiếp tục sủ̉ dụng}

Điều kiện thuận lợi được định nghĩa là mức độ mà một cá nhân tin rằng một tổ chức hoặc hạ tầng kỹ thuật hỗ trợ để sử dụng hệ thống (Venkatesh et al., 2003). Nghiên cứu của Singh và cộng sự (2017) đã chỉ ra rằng NTD sẽ có xu hướng tin tưởng vào hệ thống thương mại điện tử nếu có cơ sở hạ tầng công nghệ như Internet và tính sẵn có của các thiết bị kết nối Internet. Từ góc độ kỹ thuật, ứng dụng giao thực phẩm trên di động là một phần mềm được sử dụng trên điện thoại thông minh và không thể hoạt động hiệu quả khi không có Internet hoặc dịch vụ 3G/4G/5G. Nghiên cứu của Lee và cộng sự (2019), Alalwan (2019) kết luận rằng điều kiện thuận lợi là một trong các yếu tố tác động đến ý định tiếp tục sử dụng ứng dụng giao thực phẩm trên di động. Do đó giả thuyết $\mathrm{H} 8$ và $\mathrm{H} 9$ được đề nghị như sau:

H8: Điều kiện thuận lợi ảnh hương cùng chiều đến sụ tin tưởng ưng dụng đặt hàng thục phẩm trên di động của người tiêu dùng

H9: Điều kiện thuận lợi ảnh hưởng cùng chiều đến ý định tiếp tục sủ dụng dịch vụ giao nhận thực phẩm trên ưng dụng di động của người tiêu dùng

\section{8. Động lục thu hưởng, sụ tin tưởng và ý định tiếp tục sử dụng}

Động lực thụ hưởng được xác định như là niềm vui hay sự sung sướng có được từ việc sử dụng công nghệ mới, được chứng minh là có vai trò quan trọng trong việc sự chấp nhận và sử dụng công nghệ (Brown \& Venkatesh, 2005). Động lực thụ hưởng được xem là một yếu tố tâm lý nội tại của của mỗi khách hàng. Hwang và Kim (2007) lập luận rằng niềm vui, tâm lý của mỗi khách hàng ảnh hưởng đến niềm tin. Kết quả tương tự cũng được xác nhận bởi Akhlaq và Ahmed (2013), Sharif và Raza (2017), yếu tố động lực thụ hưởng đóng vai trò quan trọng trong việc nâng cao niềm tin của khách hàng với việc sử dụng ngân hàng điện tử. Các ứng dụng giao thực phẩm trên di động được coi là hiện đại và sáng tạo, điều này làm cho NTD cảm thấy hưng phấn và thích thú khi sử dụng các ứng dụng mới này. Nghiên cứu của Alalwan (2019) về ứng dụng giao thực phẩm tại Jordan thì NTD cảm thấy thích thú với những trải nghiệm trên ứng dụng và sẵn sàng tiếp tục sử dụng những ứng dụng này trong tương lai nếu các ứng dụng này mang lại cảm giác vui thích, thoải mái và tận hưởng. Vì vậy giả thuyết $\mathrm{H} 10$ và $\mathrm{H} 11$ được đề xuất như sau:

H10: Động lực thu hương ảnh hương cùng chiều đến sụ tin tuởng ưng dụng đặt hàng thực phẩm trên di động của người tiêu dùng

H11: Động lục thụ hương ảnh hưởng cùng chiều đến ý định tiếp tục sử dụng dịch vụ giao nhận thực phẩm trên ưng dụng di động của người tiêu dùng 


\subsection{Giá trị giá cả, sụ tin tưởng và ý định tiếp tục sủ̉ dụng}

Giá trị giá cả được hiểu là sự cân bằng nhận thức của NTD giữa lợi ích của một dịch vụ mang lại và chi phí tiền tệ để sử dụng chúng (Venkatesh et al., 2012). Giá trị giá cả ảnh hưởng tích cực khi NTD nhận thấy lợi ích họ nhận được nhiều hơn chi phí mà họ phải bỏ ra. Nghiên cứu hành vi NTD của Garbarino và Lee (2003) cho thấy rằng sự không công bằng giữa khoản tiền mà NTD chi ra so với lợi ích mà họ nhận được từ sản phẩm/dịch vụ sẽ ảnh hưởng tiêu cực đối với niềm tin của NTD và ý định mua lặp lại sản phẩm/dịch vụ tại website bán lẻ trực tuyến. Nghiên cứu của Rahi, Ghani, và Muhamad (2017) chỉ ra rằng nhận thức về giá cả hợp lý ảnh hưởng tích cực đến sự tin tưởng khi mua sắm trực tuyến. Theo Venkatesh và cộng sự (2012), giá trị giá cả là một trong những yếu tố mạnh ảnh hưởng đến việc tiếp tục sử dụng dịch vụ Internet trên di động. Hơn nữa, nghiên cứu của Alalwan (2019) cho thấy giá cả ảnh hưởng tích cực đến ý định sử dụng tiếp tục ứng dụng đặt hàng thực phẩm trên di động. Do đó giả thuyết $\mathrm{H} 12$ và H13 được đề xuất như sau:

H12: Giá trị giá cả ảnh hưởng cùng chiều đến sụ tin tương ứng dụng đặt hàng thụcc phẩm trên di động của người tiêu dùng

H13: Giá trị giá cả ảnh hương cùng chiều đến ý định tiếp tục sử dụng dịch vu giao nhận thục phẩm trên ưng dụng di động của người tiêu dùng

\subsection{Thói quen, sụ tin tưởng và ý định tiếp tục sủ̉ dụng}

Thói quen được xác định là xu hướng được thực hiện một cách tự động thông qua các hành động được lặp đi lặp lai nhiều lần trong quá khứ. Venkatesh và cộng sự (2012) cho rằng việc sử dụng kinh nghiệm trước đó là điều kiện tiên quyết cho thói quen sử dụng công nghệ và thói quen đó là yếu tố chính trong việc chấp nhận công nghệ đó trong tương lai. Nghiên cứu của Liao, Palvia, và Lin (2006) về thói quen trong giao dịch thương mại điện tử cũng cho rằng bên cạnh nhận thức về tính hữu dụng và sự tin tưởng được coi là yếu tố dự báo chính của ý định sử dụng, một đóng góp đáng kể của nghiên cứu này là xác nhận tầm quan trọng của yếu tố thói quen của NTD trong giao dịch thương mại điện tử. Thói quen không chỉ ảnh hưởng tích cực đến nhận thức tính hữu dụng và ý định tiếp tục sử dụng mà còn tác động tích cực đến sự tin tưởng của NTD khi mua sắm trực tuyến. Ngày nay, mọi người ngày càng gắn bó với điện thoại thông minh và hình thành thói quen sử dụng điện thoại hằng ngày để mua sắm. Alalwan (2019) và Lee và cộng sự (2019) đã nhận thấy rằng khách hàng có thói quen sử dụng ứng dụng giao thực phẩm trên thiết bị di động và thói quen này đủ lớn sẽ ảnh hưởng tích cực đến ý định tái sử dụng ứng dụng trong tương lai. Từ lập luận trên, giả thuyết $\mathrm{H} 14$ và $\mathrm{H} 15$ được đề xuất như sau:

H14: Thói quen ảnh hưởng cùng chiều đến sụ tin tưởng ứng dụng đặt hàng thực phẩm trên di động của người tiêu dùng

H15: Thói quen ảnh huởng cùng chiều đến ý định tiếp tục sủ dụng dịch vu giao nhận thực phẩm trên úng dụng di động của người tiêu dùng

\subsection{Chất lự̛ng thông tin, sụ tin tưởng và ý định tiếp tục sử dụng}

Chất lượng thông tin là nhận thức của NTD về mức độ mà thông tin được cung cấp được cho là chính xác, phù hợp với định dạng nhất định và có nội dung được cập nhật (Kuan, Bock, \& Vathanophas, 2008). Theo nghiên cứu của Liao và cộng sự (2006) thì chất lượng thông tin của trang mua sắm trực tuyến càng cao thì khách hàng càng tin tưởng khi mua sản phẩm trực tuyến. Nicolaou và McKnight (2006) cũng khẳng định chất lượng thông tin trên website có ảnh hưởng sâu sắc đến mức độ cảm nhận rủi ro và sự tin tưởng của NTD. Các nghiên cứu của Panse, Rastogi, Sharma, và Dorji (2019), Lee và cộng sự (2019) cho thấy chất lượng thông tin mô tả 
thực phẩm càng cao thì NTD càng tin tưởng và từ đó đưa ra các ý định mua hàng khác nhau. Dựa vào các đánh giá thực nghiệm trên, giả thuyết $\mathrm{H} 16$ và $\mathrm{H} 17$ được đề nghị là:

H16: Chất luợng thông tin ảnh hưởng cùng chiều đến sụ tin tuởng ưng dụng đặt hàng thực phẩm trên di động của người tiêu dùng

H17: Chất lượng thông tin ảnh hương cùng chiều đến ý định tiếp tục sủ dụng dịch vu giao nhận thực phẩm trên ưng dụng di động của người tiêu dùng

Dựa trên cơ sở lý thuyết và các giả thuyết nghiên cứu đã đề xuất, một mô hình nghiên cứu được hình thành bao gồm 17 mối quan hệ thể hiện các biến độc lập gồm có: kỳ vọng hiệu quả, kỳ vọng nỗ lực, ảnh hưởng xã hội, điều kiện thuận lợi, động lực thụ hưởng, giá trị giá cả, thói quen và chất lượng thông tin vừa tác động trực tiếp và gián tiếp đến biến phụ thuộc ý định tiếp tục sử dụng thông qua biến trung gian sự tin tưởng của NTD về dịch vụ giao hàng thực phẩm thông qua UDDĐ. Hình 1 thể hiện mô hình nghiên cứu được đề xuất.

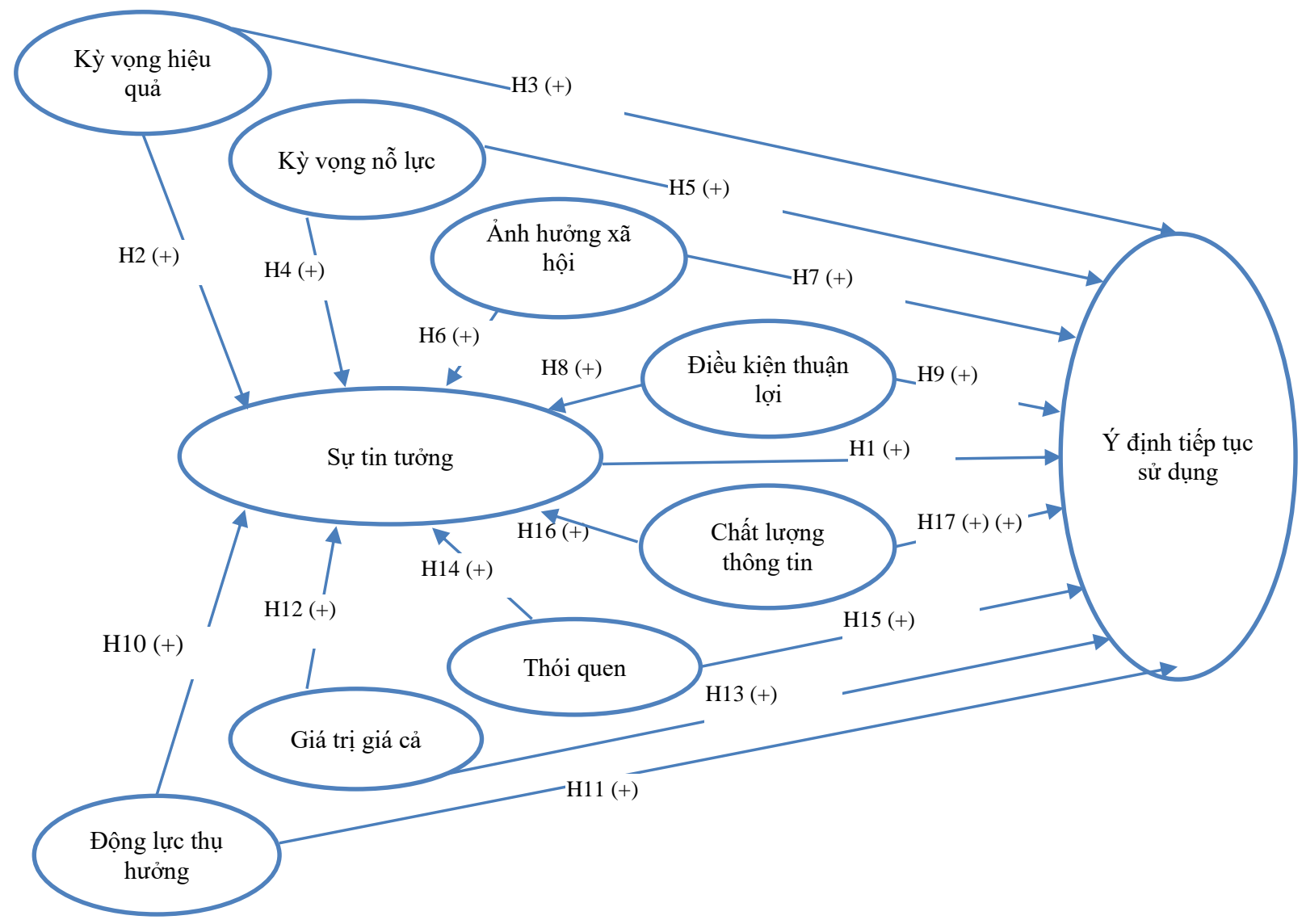

Hình 1. Mô hình nghiên cứu được đề xuất

\section{Phương pháp nghiên cứu}

Nghiên cứu này trải qua hai bước nghiên cứu định tính và nghiên cứu định lượng. Phương pháp nghiên cứu định tính thể hiện cụ thể bằng phương pháp phỏng vấn tay đôi bán cấu trúc với 10 NTD sinh sống tại Thành phố Hồ Chí Minh và đã từng sử dụng ứng dụng dịch vụ giao hàng thực phẩm trên đi động. Thành phố Hồ Chí Minh là giới hạn địa lý được chọn vì là thành phố thương mại lớn nhất Việt Nam với số lượng người dùng Internet, dùng điện thoại thông minh chiếm tỉ lệ cao nhất với hơn $38 \%$ số lượng người tiêu dùng mua sắm hàng hóa trên di động tại Việt Nam (Appota, 2018). 
Kết quả nghiên cứu định tính cho thấy có 08 biến quan sát được điều chỉnh để dễ hiểu hơn và bổ sung thêm 03 biến quan sát mới cho các thang đo trong nghiên cứu định lượng. Phiếu khảo sát định lượng được xây dựng gồm ba phần: (1) câu hỏi sàng lọc, (2) câu hỏi chính với 42 biến quan sát sử dụng thang đo Likert từ $1-5$ cho 10 yếu tố tiềm ẩn và (3) câu hỏi về nhân khẩu học. Phương pháp chọn mẫu phi xác suất thuận tiện được sử dụng với đối tượng khảo sát được sàng lọc là những người đã từng sử dụng các ứng dụng giao hàng thực phẩm (như Grabfood, Foody, Go-Food, Baemin, Ahamove, Loship). Cách tiếp cận đối tượng khảo sát thông qua việc phát phiếu khảo sát trực tiếp tại điểm tiếp cận (nhà ở, trường học, nơi làm việc) và khảo sát trực tuyến. Với tổng số phiếu khảo sát được phát đi là 536 phiếu khảo sát, tổng số phiếu khảo sát thu được là 392. Sau khi kiểm tra sàng lọc thu được 378 phiếu khảo sát hợp lệ có thể được dùng cho xử lý và phân tích dữ liệu thống kê, từ đó sản sinh ra kết quả nghiên cứu có ý nghĩa đáng kể như sau đây.

\section{Kết quả nghiên cứu}

\section{1. Đặc điểm mẫu nghiên cúu}

Đối tượng khảo sát là nam chiếm tỉ lệ $39.7 \%$ và nữ chiếm tỉ lệ $60.3 \%$. Nhóm tuổi trẻ từ 20 đến 30 tuổi là đối tượng sử dụng nhiều nhất $(62.4 \%)$, tiếp theo là nhóm tuổi từ $31-40$ tuổi (27.8\%), nhóm tuổi dưới 20 và nhóm tuổi trên 40 tuổi chiếm tỉ lệ thấp, khoảng $10 \%$. Khoảng $2 / 3$ (66.1\%) nhóm đối tượng khảo sát đang ở tình trạng độc thân, và khoảng $1 / 3$ nhóm người còn lại đã lập gia đình (33.9\%). Hơn 70\% đối tượng khảo sát có trình độ đại học và sau đại học, tiếp theo đó là Cao đẳng $(12.7 \%)$, Trung cấp $(8.2 \%)$ và trình độ khác $(8.5 \%)$. Gần $1 / 2$ mẫu nghiên cứu có thu nhập hàng tháng dưới 10 triệu đồng (49.3\%), số người có thu nhập từ 10 - 15 triệu trở lên chiếm gần $1 / 4$ mẫu nghiên cứu (24.1\%), số người có mức thu nhập cao hơn 20 triệu chiếm tỉ lệ thấp (12.9\%). Gần một nửa mẫu nghiên cứu đã từng sử dụng ứng dụng dịch vụ đặt thực phẩm trên một năm và một nửa còn lại chỉ mới bắt đầu sử dụng gần đây. Phần lớn NTD sử dụng với tần suất sử dụng từ 01 - 04 lần/tháng $(57.4 \%)$, nhóm đối tượng sử dụng trên 08 lần/tháng cũng tương đối $(18.5 \%)$. Phần lớn khách hàng đều cài đặt trên hai ứng dụng đặt hàng thực phẩm để sử dụng $(64.8 \%)$. Trong đó ứng dụng Now được cài đặt trên điện thoại nhiều nhất $(29.4 \%)$, tiếp theo là GrabFood (21.2\%), Foody (17.4\%), Go-Food (14.9\%), Baemin (12.4\%), Ahavove (3.1\%), Loship $(0.5 \%)$ và khác $1.2 \%$. Việc cài đặt nhiều ứng dụng trên điện thoại di động cho phép NTD có thể tìm kiếm các chương trình khuyến mãi trên các ứng dụng khác nhau khi sử dụng.

\section{2. Đánh giá giá trị hội tụ của thang đo}

Kết quả chạy bằng phần mềm SPSS với hệ số Cronbach' Aplpha lần lượt của các thang đo có giá trị dao động thấp nhất là 0.774 của nhân tố "Ảnh hưởng xã hội” và cao nhất là 0.914 của nhân tố "Ý định tiếp tục sử dụng” (đáp ứng Cronbach’s Alpha $\geq 0.7$, Hair, Black, Babin, \& Aderson, 2014). Đồng thời, giá trị tương quan biến tổng của từng biến quan sát đều lớn hơn 0.3 và không có biến quan sát nào khi loại sẽ có kết quả lớn hơn so với Cronbach’ Alpha tổng. Kết quả phân tích nhân tố khám phá EFA được thực hiện chạy 02 lần với loại bỏ lần lượt các biến $\mathrm{PE} 4$ và $\mathrm{PV} 3$ vì hệ số tải nhân tố của chúng nhỏ hơn 0.5 . Bảng 1 trình bày bảng tổng hợp kết quả kiểm định với hệ số tải đã chuẩn hóa dao động từ 0.578 đến 0.887 (đáp ứng điều kiện lớn hơn 0.5, Hair et al., 2014), hệ số tin cậy tổng hợp (CR) dao động từ 0.754 đến 0.915 (đáp ứng điều kiện $C R>0.7)$ và phương sai trích trung bình $(\mathrm{AVE})$ của các thang đo dao động từ 0.506 đến 0.728 (đáp ứng điều kiện $\mathrm{AVE}>0.5$ ). Do vậy, có thể kết luận rằng các thang đo đảm bảo tốt nhất giá trị hội tụ. 


\section{Bảng 1}

Kết quả kiểm định Cronbach’ Alpha và phân tích nhân tố khám phá

\begin{tabular}{|c|c|c|c|c|}
\hline $\begin{array}{c}\text { Mã } \\
\text { biến }\end{array}$ & Các nhận định & $\begin{array}{l}\text { Hệ số tải } \\
\text { nhân tố }\end{array}$ & $\mathbf{C R}$ & AVE \\
\hline \multicolumn{5}{|c|}{ Kỳ vọng hiệu quả (Cronbach's $\alpha=0.825$ ) } \\
\hline PE1 & $\begin{array}{l}\text { Tôi thấy ứng dụng đặt hàng thực phẩm trên di động hữu } \\
\text { ích cho cuộc sống hằng ngày. }\end{array}$ & .730 & \multirow{4}{*}{0.817} & \multirow{4}{*}{0.529} \\
\hline PE2 & $\begin{array}{l}\text { Đặt hàng thực phẩm trên UDDĐ giúp tôi làm được nhiều } \\
\text { việc quan trọng hơn trong khi chờ đợi. }\end{array}$ & .787 & & \\
\hline PE3 & $\begin{array}{l}\text { Đặt hàng thực phẩm trên UDDĐ giúp cho tôi mua hàng } \\
\text { nhanh chóng hơn. }\end{array}$ & .681 & & \\
\hline PE5 & $\begin{array}{l}\text { Tôi có thể tiết kiệm thời gian khi đặt hàng thực phẩm trên } \\
\text { UDDĐ. }\end{array}$ & 698 & & \\
\hline \multicolumn{5}{|c|}{ Kỳ vọng nỗ lực (Cronbach's $\alpha=0.855)$} \\
\hline EE1 & Tôi dễ dàng học cách đặt hàng thực phẩm trên UDDĐ. & .728 & \multirow{4}{*}{0.856} & \multirow{4}{*}{0.598} \\
\hline EE2 & $\begin{array}{l}\text { Úng dụng đặt hàng thực phẩm rõ ràng và dễ hiểu giúp tôi } \\
\text { dễ tương tác. }\end{array}$ & .864 & & \\
\hline EE3 & Tôi thấy ứng dụng đặt hàng thực phẩm dễ sử dụng. & .719 & & \\
\hline EE4 & $\begin{array}{l}\text { Tôi dễ dàng sử dụng thuần thục cách đặt hàng thực phẩm } \\
\text { trên U'DDĐ. }\end{array}$ & .701 & & \\
\hline
\end{tabular}

\section{Ảnh hưởng xã hội (Cronbach's $\alpha=0.774)$}

SI1 Những người quan trọng với tôi cho rằng tôi nên sử dụng UDDĐ để đặt thực phẩm.

SI2 Những người ảnh hưởng đến hành vi của tôi nghĩ rằng tôi nên sử dụng UDDĐ để đặt hàng thực phẩm.

$\begin{array}{lll}.709 & 0.774 & 0.535\end{array}$

SI3 Những người xung quanh đánh giá cao việc tôi sử dụng ứng di động để đặt hàng thực phẩm.

.682

Điều kiện thuận lợi (Cronbach's $\alpha=0.830)$

FC1 Thiết bị của tôi có khả năng cài đặt và kết nối với ứng dụng để đặt hàng thực phẩm.

FC2 Tôi có các kiến thức cần thiết để đặt hàng thực phẩm trên UDDĐ.

FC3 Úng dụng đặt hàng thực phẩm tương tự với các ứng dụng khác mà tôi đang sử dụng trên di động.

FC4 Có đường dây hỗ trợ nếu tôi gặp khó khăn khi sử dụng ứng dụng đặt hàng thực phẩm.

\section{Động lực thụ hưởng (Cronbach's $\alpha=\mathbf{0 . 8 8 5}$ )}

HM1 Tôi thấy hào hứng khi sử dụng ứng dụng đặt hàng thực phẩm trên di động.

HM2 Sử dụng UDDĐ để đặt hàng thực phẩm mang đến những trải nghiệm thú vị.

HM3 Sử dụng UDDĐ để đặt hàng thực phẩm mang tính chất giải trí.

PV1 Đặt hàng thực phẩm trên ƯDDĐ có giá hợp lý.

PV2 Tôi nhận được nhiều lơi ích hơn so với tiền bỏ ra khi sử dụng U’DDĐ để đặt thực phẩm. 


\begin{tabular}{|c|c|c|c|}
\hline $\begin{array}{l}\text { Mã } \\
\text { biến }\end{array}$ & Các nhận định & $\begin{array}{l}\text { Hệ số tải } \\
\text { nhân tố }\end{array}$ & CR \\
\hline PV4 & $\begin{array}{l}\text { Tôi được lợi về các khuyến mãi khi đặt hàng thực phẩm } \\
\text { trên UDDĐ. }\end{array}$ & .729 & \\
\hline
\end{tabular}

\begin{tabular}{|c|c|c|c|c|}
\hline \multicolumn{5}{|c|}{ Thói quen (Cronbach's $\alpha=0.808$ ) } \\
\hline HB1 & $\begin{array}{l}\text { Đă̆t hàng thực phẩm trên U'DDĐ gần như là một thói quen } \\
\text { đối với tôi. }\end{array}$ & .634 & \multirow{4}{*}{\multicolumn{2}{|c|}{0.8120 .523}} \\
\hline HB2 & $\begin{array}{l}\text { Tôi nghiện sử dụng ứng dụng đặt hàng thực phẩm trên di } \\
\text { động. }\end{array}$ & .787 & & \\
\hline HB3 & Tôi luôn đặt hàng thực phẩm trên U’DDĐ khi cần. & .803 & & \\
\hline HB4 & Tôi cảm thấy quen thuộc khi đặt thực phẩm trên ƯDDĐ. & .644 & & \\
\hline \multicolumn{5}{|c|}{ Chất lượng thông tin (Cronbach's $\alpha=0.851$ ) } \\
\hline IQ1 & $\begin{array}{l}\text { Ú́ng dụng đặt hàng thực phẩm cung cấp thông tin chính } \\
\text { xác. }\end{array}$ & .703 & \multirow{5}{*}{0.853} & \multirow{5}{*}{0.538} \\
\hline IQ2 & $\begin{array}{l}\text { Úng dụng đặt hàng thực phẩm cung cấp thông tin đáng tin } \\
\text { cậy. }\end{array}$ & .831 & & \\
\hline IQ3 & $\begin{array}{l}\text { Úng dụng đặt hàng thực phẩm cung cấp thông tin rất chi } \\
\text { tiêt. }\end{array}$ & .829 & & \\
\hline IQ4 & $\begin{array}{l}\text { Úng dụng đặt hàng thực phẩm cung cấp thông tin rất rõ } \\
\text { ràng. }\end{array}$ & .681 & & \\
\hline IQ5 & $\begin{array}{l}\text { Úng dụng đặt hàng thực phẩm trình bày nội dung rất dễ } \\
\text { hiểu. }\end{array}$ & .601 & & \\
\hline
\end{tabular}

\section{Sự tin tưởng (Cronbach's $\alpha=\mathbf{0 . 8 8 9})$}

TR1 Tôi tin rằng ứng dụng đặt hàng thực phẩm là đáng tin cậy.

TR2 Tôi tin rằng ứng dụng đặt hàng thực phẩm thực hiện đúng cam kết.

TR3 Tôi không lo lắng khi sử dụng ứng dụng đặt hàng thực phẩm.

TR4 Ngay cả khi không theo dõi đơn hàng, tôi vẫn tin rằng ứng dụng đặt hàng thực phẩm sẽ thực hiện đúng yêu cầu của tôi.

$0.892 \quad 0.582$

TR5 Tôi không nghi ngờ về tính trung thực của ứng dụng đặt hàng thực phẩm.

TR6 Tôi tin rằng ứng dụng đặt hàng thực phẩm dựa trên nhu cầu của người tiêu dùng.

.702

\section{Ý định tiếp tục sử dụng (Cronbach's $\alpha=0.914)$}

CI1 Tôi có ý định đặt hàng lại thực phẩm trên U'DDĐ trong tương lai.

CI2 Tôi sẽ sử dụng lại ƯDDĐ để đặt thực phẩm trong cuộc sống hằng ngày.

CI3 Tôi có kế hoạch tiếp tục đặt hàng thực phẩm trên U'DDĐ thường xuyên hơn.

CI4 Tôi đã quyết định đặt hàng thực phẩm trên U’DDĐ ở lần kế tiếp.

$\mathrm{KMO}=0.915$; Kiểm định Barlett's có sig = 0.000; Eigenvalues = 1.016; Tổng phương sai trích $=59.674 \%$. 


\section{3. Đánh giá giá trị phân biệt của thang đo}

Bảng 2 thể hiện kết quả kiểm định giá trị phân biệt, với giá trị in đậm là căn bậc hai của AVE lớn hơn các giá trị dưới đường chéo là hệ số tương quan giữa hai khái niệm tương ứng, đồng thời giá trị của các khái niệm đều có $\mathrm{MSV}<\mathrm{AVE}$. Do đó có thể kết luận rằng thang đo của các khái niệm trong mô hình có tính phân biệt với nhau (Hu \& Bentler, 1999).

\section{Bảng 2}

Kết quả kiểm định giá trị phân biệt

\begin{tabular}{lcccccccccccc}
\hline & AVE & MSV & TR & IQ & EE & HB & PE & HM & FC & SI & CI & PV \\
\hline TR & 0.582 & 0.522 & $\mathbf{0 . 7 6 3}$ & & & & & & & & & \\
IQ & 0.538 & 0.335 & 0.558 & $\mathbf{0 . 7 3 4}$ & & & & & & & & \\
EE & 0.598 & 0.417 & 0.646 & 0.453 & $\mathbf{0 . 7 7 3}$ & & & & & & & \\
HB & 0.523 & 0.103 & 0.286 & 0.247 & 0.255 & $\mathbf{0 . 7 2 4}$ & & & & & & \\
PE & 0.529 & 0.252 & 0.498 & 0.363 & 0.399 & 0.267 & $\mathbf{0 . 7 2 7}$ & & & & & \\
HM & 0.724 & 0.213 & 0.361 & 0.334 & 0.257 & 0.156 & 0.336 & $\mathbf{0 . 8 5 1}$ & & & & \\
FC & 0.553 & 0.311 & 0.543 & 0.440 & 0.478 & 0.189 & 0.261 & 0.271 & $\mathbf{0 . 7 4 4}$ & & & \\
SI & 0.535 & 0.323 & 0.483 & 0.425 & 0.420 & 0.242 & 0.196 & 0.317 & 0.432 & $\mathbf{0 . 7 3 1}$ & & \\
CI & 0.728 & 0.522 & 0.722 & 0.579 & 0.642 & 0.321 & 0.502 & 0.461 & 0.558 & 0.568 & $\mathbf{0 . 8 5 4}$ & \\
PV & 0.506 & 0.319 & 0.549 & 0.380 & 0.519 & 0.108 & 0.282 & 0.220 & 0.372 & 0.257 & 0.565 & $\mathbf{0 . 7 1 1}$ \\
\hline
\end{tabular}

PE: kỳ vọng hiệu quả, EE: kỳ vọng nỗ lực, SI: ảnh hưởng xã hội, FC: điều kiện thuận lợi, HM: động lực thụ hưởng, PV: giá trị giá cả, HB: thói quen, IQ: chất lượng thông tin, TR: sự tin tưởng, $\mathbf{C I}$ : ý định tiếp tục sử dụng.

Nguồn: Kết quả phân tích dữ liệu của nhóm nghiên cứu (2020)

\subsection{Kiểm định các giả thuyết và mô hình nghiên cứu}

Mô hình thống kê cấu trúc SEM được dùng để có được kết quả kiểm định mô hình nghiên cứu đã đề xuất. Kết quả cho thấy mô hình nghiên cứu phù hợp với dữ liệu nghiên cứu thị trường thông qua các chỉ số Chi-square/df $=1.396, \mathrm{P}$-value $=0.000, \mathrm{RMSEA}=0.032, \mathrm{CFI}=$ $0.964, \mathrm{TLI}=0.960, \mathrm{SRMR}=0.0394$. Bảng 3 cho thấy có ba giả thuyết H10, H14 và H15 bị bác bỏ vì có giá trị $p>0.05$. Các giả thuyết còn lại (14 giả thuyết) đều được chấp nhận vì có giá trị $p$ $<0.05$ và có hệ số chuẩn hóa đều dương nên có tác động cùng chiều, phù hợp với chiều của các giả thuyết đã được đề xuất trong mô hình nghiên cứu.

\section{Bảng 3}

Kết quả hệ số mô hình hồi quy chuẩn hóa SEM

\begin{tabular}{ccccccccc}
\hline \multicolumn{2}{c}{ Mối quan hệ } & $\begin{array}{c}\text { Giả } \\
\text { thuyết }\end{array}$ & $\begin{array}{c}\text { Hệ số hồi quy đã } \\
\text { chuẩn hóa }\end{array}$ & S.E. & C.R. & Giá trị p & $\begin{array}{c}\text { Kiểm định } \\
\text { giả thuyết }\end{array}$ \\
\hline $\mathrm{TR}$ & $\rightarrow$ & $\mathrm{CI}$ & $\mathrm{H} 1$ & 0.185 & 0.064 & 2.835 & 0.005 & Chấp nhận \\
$\mathrm{PE}$ & $\rightarrow$ & $\mathrm{TR}$ & $\mathrm{H} 2$ & 0.197 & 0.048 & 3.858 & $* * *$ & Chấp nhận \\
$\mathrm{PE}$ & $\rightarrow$ & $\mathrm{CI}$ & $\mathrm{H} 3$ & 0.136 & 0.044 & 2.864 & 0.004 & Chấp nhận \\
$\mathrm{EE}$ & $\rightarrow$ & $\mathrm{TR}$ & $\mathrm{H} 4$ & 0.239 & 0.044 & 3.939 & $* * *$ & Chấp nhận
\end{tabular}




\begin{tabular}{|c|c|c|c|c|c|c|c|c|}
\hline \multicolumn{3}{|c|}{ Mối quan hệ } & \multirow{2}{*}{$\begin{array}{c}\begin{array}{c}\text { Giả } \\
\text { thuyết }\end{array} \\
\text { H5 }\end{array}$} & \multirow{2}{*}{$\begin{array}{c}\begin{array}{c}\text { Hệ số hồi quy đã } \\
\text { chuẩn hóa }\end{array} \\
0.14\end{array}$} & \multirow{2}{*}{$\begin{array}{l}\text { S.E. } \\
0.04\end{array}$} & \multirow{2}{*}{$\begin{array}{l}\text { C.R. } \\
2.515\end{array}$} & \multirow{2}{*}{$\begin{array}{c}\text { Giá trị p } \\
0.012\end{array}$} & \multirow{2}{*}{$\begin{array}{l}\begin{array}{l}\text { Kiểm định } \\
\text { giả thuyết }\end{array} \\
\text { Chấp nhận }\end{array}$} \\
\hline $\mathrm{EE}$ & $\rightarrow$ & CI & & & & & & \\
\hline SI & $\rightarrow$ & TR & H6 & 0.136 & 0.058 & 2.479 & 0.013 & Chấp nhận \\
\hline SI & $\rightarrow$ & CI & $\mathrm{H} 7$ & 0.199 & 0.053 & 3.896 & $* * *$ & Chấp nhận \\
\hline $\mathrm{FC}$ & $\rightarrow$ & TR & $\mathrm{H} 8$ & 0.156 & 0.047 & 2.903 & 0.004 & Chấp nhận \\
\hline FC & $\rightarrow$ & CI & H9 & 0.107 & 0.042 & 2.183 & 0.029 & Chấp nhận \\
\hline HM & $\rightarrow$ & TR & H10 & 0.047 & 0.034 & 1.024 & 0.306 & Bác bỏ \\
\hline HM & $\rightarrow$ & CI & H11 & 0.137 & 0.03 & 3.334 & $* * *$ & Chấp nhận \\
\hline PV & $\rightarrow$ & TR & H12 & 0.206 & 0.047 & 3.62 & $* * *$ & Chấp nhận \\
\hline PV & $\rightarrow$ & CI & $\mathrm{H} 13$ & 0.187 & 0.044 & 3.505 & $* * *$ & Chấp nhận \\
\hline HB & $\rightarrow$ & TR & H14 & 0.045 & 0.052 & 0.998 & 0.318 & Bác bỏ \\
\hline HB & $\rightarrow$ & CI & H15 & 0.062 & 0.046 & 1.532 & 0.125 & Bác bỏ \\
\hline IQ & $\rightarrow$ & TR & H16 & 0.147 & 0.047 & 2.729 & 0.006 & Chấp nhận \\
\hline IQ & $\rightarrow$ & CI & H17 & 0.1 & 0.041 & 2.04 & 0.041 & Chấp nhận \\
\hline
\end{tabular}

Nguồn: Kết quả phân tích dữ liệu của nhóm nghiên cứu (2020)

\section{5. Đánh giá mức độ ảnh huơong gián tiếp và trục tiếp đến ý định tiếp tục sử dụng}

Bảng 4 cho thấy các yếu tố kỳ vọng hiệu quả, kỳ vọng nỗ lực, ảnh hưởng xã hội, điều kiện thuận lợi, giá trị giá cả, chất lượng thông tin có ảnh hưởng trực tiếp và gián tiếp đến ý định tiếp tục sử dụng thông qua biến trung gian là sự tin tưởng.

\section{Bảng 4}

Tác động trực tiếp và gián tiếp đến ý định tiếp tục sử dụng (đã chuẩn hóa)

\begin{tabular}{|c|c|c|c|}
\hline \multirow[b]{2}{*}{ Mối quan hệ } & \multicolumn{3}{|c|}{ Hệ số beta chuẩn hóa } \\
\hline & Ảnh hưởng trực tiếp & Ảnh hưởng gián tiếp & $\begin{array}{c}\text { Ảnh hưởng tổng } \\
\text { hợp }\end{array}$ \\
\hline Kỳ vọng hiệu quả & 0.136 & 0.036 & 0.172 \\
\hline Kỳ vọng nỗ lực & 0.140 & 0.044 & 0.184 \\
\hline Ảnh hưởng xã hội & 0.199 & 0.025 & 0.224 \\
\hline Điều kiện thuận lợi & 0.107 & 0.029 & 0.136 \\
\hline Động lực thụ hưởng & 0.137 & 0.009 & 0.146 \\
\hline Giá trị giá cả & 0.187 & 0.038 & 0.225 \\
\hline Chất lượng thông tin & 0.100 & 0.027 & 0.127 \\
\hline Sự tin tưởng & 0.185 & 0.000 & 0.185 \\
\hline
\end{tabular}

Nguồn: Kết quả xử lý từ dữ liệu điều tra (2020) 
Theo bảng trên, nếu tính gộp cả tác động trực tiếp và gián tiếp thì mức độ ảnh hưởng đáng kể của các yếu tố chính đến ý định tiếp tục sử dụng dịch vụ giao thực phẩm trên di động có khác nhau. Cụ thể theo thứ tự từ cao xuống thấp là: giá trị giá cả $(0.225)$, ảnh hưởng xã hội (0.224), sự tin tưởng (0.185), kỳ vọng nỗ lực (0.184), kỳ vọng hiệu quả (0.172), động lực thụ hưởng $(0.146)$, và chất lượng thông tin $(0.127)$.

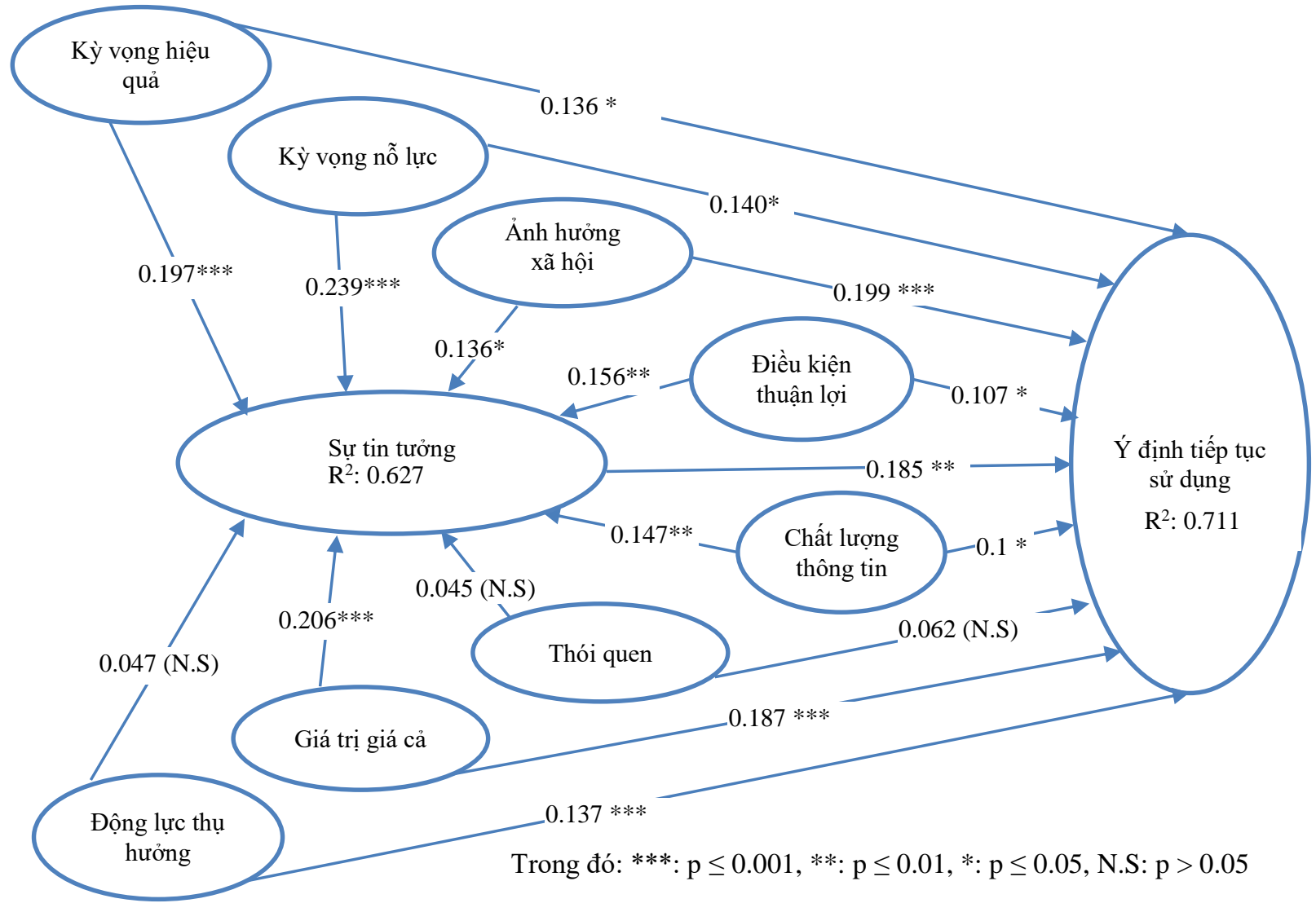

Hình 2. Kết quả kiểm định mô hình nghiên cứu

Hình 2 kết luận tổng thể các giả thuyết nghiên cứu sau kiểm định thống kê. Kết quả cho thấy yếu tố thói quen bị loại ra khỏi mô hình nghiên cứu vì thói quen không ảnh hưởng đáng kể đến sự tin tưởng và ý định tiếp tục sử dụng (bác bỏ giả thuyết H14 và H15). Biến động lực thụ hưởng mặc dù không ảnh hưởng đáng kể đến biến sự tin tưởng nhưng có ảnh hưởng tương đối lớn đến biến ý định tiếp tục sử dụng (bác bỏ giả thuyết $\mathrm{H} 10$ và chấp nhận giả thuyết $\mathrm{H} 11$ ). Hệ số hồi quy bội $\mathrm{R}^{2}$ cho thấy có thể giải thích được $62.7 \%$ sự biến thiên của biến sự tin tưởng và $71.1 \%$ sự biến thiên của biến ý định tiếp tục sử dụng dựa trên các biến độc lập của mô hình nghiên cứu. Sự tác động trực tiếp của biến sự tin tưởng đối với ý định tiếp tục sử dụng là tương đối lớn. Đồng thời với vai trò là biến trung gian, sự tin tưởng đã làm cho các yếu tố chính tác động đến ý định tiếp tục sử dụng mạnh hơn so với các tác động trực tiếp riêng lẻ.

\section{Kết luận và thảo luận kết quả}

Kết quả nghiên cứu đã chỉ ra được có 08 yếu tố có tác động đến ý định tiếp tục sử dụng ứng dụng đặt hàng thực phẩm gồm có: kỳ vọng hiệu quả, kỳ vọng nỗ lực, ảnh hưởng xã hội, điều kiện thuận lợi, động lực thụ hưởng, giá trị giá cả, chất lượng thông tin và sự tin tưởng. Đồng thời kết quả cũng cho thấy có 06 yếu tố cũng tác động tích cực đến sự tin tưởng của NTD đối với ứng dụng gồm có: kỳ vọng hiệu quả, kỳ vọng nỗ lực, ảnh hưởng xã hội, điều kiện thuận lợi, giá trị giá cả và chất lượng thông tin. 
Sự tin tưởng là một yếu tố tác động tích cực đối với ý định tiếp tục sử dụng, đây là cũng là nét tương đồng đối với các phần lớn các ứng dụng di động và dịch vụ thương mại điện tử. Khi NTD càng tin tưởng vào các ứng dụng di động và các dịch vụ thương mại trực tuyến, họ sẽ càng tin tưởng và có ý định tiếp tục sử dụng ứng dụng và dịch vụ đó. Ngoài tác động trực tiếp của sự tin tưởng đến ý định tiếp tục sử dụng thì yếu tố sự tin tưởng cũng là biến trung gian của sự tác động của các yếu tố khác đối với ý định tiếp tục sử dụng. Trong đó, hai yếu tố giá trị giá cả và ảnh hưởng xã hội là hai yếu tố mạnh nhất tác động tổng hợp trực tiếp và gián tiếp tới đến ý định tiếp tục sử dụng thông qua biến trung gian sự tin tưởng. Điều này có nghĩa là khi NTD đạt được nhiều lợi ích về giá và được nhiều người thân, bạn bè giới thiệu và khuyên dùng thì sẽ càng tin tưởng và từ đó sẽ càng tiếp tục sử dụng ứng dụng này trong tương lai.

Kết quả nghiên cứu cũng cho thấy sự ảnh hưởng tích cực của các yếu tố kỳ vọng hiệu quả, kỳ vọng nỗ lực, điều kiện thuận lợi và chất lượng thông tin đến sự tin tưởng và ý định tiếp tục sử dụng ứng dụng. NTD đã rất quan tâm đến các lợi ích gia tăng do dịch vụ mang lại bao gồm sự hữu ích cho cuộc sống hằng ngày, làm được nhiều việc trong khi chờ đợi, mua hàng nhanh chóng và tiết kiệm thời gian. Những lợi ích này góp phần nâng cao niềm tin của NTD đối với ứng dụng, thúc đẩy hành vi tiếp tục sử dụng ứng dụng. Sự chấp nhận tác động của yếu tố kỳ vọng nỗ lực cho thấy các ứng dụng hiện nay được thiết kế dễ hiểu, dễ sử dụng, điều này góp phần giúp NTD tin tưởng và sử dụng tiếp tục ứng dụng này trong tương lai. Khi điều kiện thuận lợi phát huy tác dụng như có thiết bị kết nối Internet dễ dàng, có chức năng tương tự với các ứng dụng khác và có đường dây nóng hỗ trợ khi cần thiết sẽ giúp cho NTD tin tưởng hơn và tiếp tục sử dụng dịch vụ này. Chất lượng thông tin trên các ứng dụng giao thực phẩm biểu hiện qua sự chính xác, tin cậy, chi tiết, rõ ràng và dễ hiểu góp phần đáng kể đến việc củng cố niềm tin và hướng đến việc sử dụng tiếp tục ứng dụng. Điều này hoàn toàn hợp lý khi thực phẩm trên ứng dụng là một sản phẩm khó có thể đánh giá qua các thông số kỹ thuật hay khó so sánh về chất lượng. Do vậy, thông tin càng chi tiết về hình ảnh, miêu tả rõ về thành phần thực phẩm, cũng như các chỉ số đánh giá tốt/xấu của NTD đã dùng sẽ góp phần làm tăng mức độ tin cậy và quyết định sử dụng tiếp tục ứng dụng này trong tương lai.

Động lực thụ hưởng tác động tích cực đến ý định tiếp tục sử dụng ứng dụng mặc dù không thông qua sự tin tưởng đối với ứng dụng này. Rõ ràng sự tác động tích cực của niềm vui, sự hào hứng và những trải nghiệm thú vị của NTD kích thích mạnh đến hành vi tiếp tục sử dụng ứng dụng và bỏ qua yếu tố rủi ro có thể xảy ra. Kết quả này tương đồng với kết quả nghiên cứu của Giovannini, Ferreira, Silva, và Ferreira (2015), Alalwan (2019) và khác biệt so với kết quả nghiên cứu của Akhlaq và Ahmed (2013), Sharif và Raza (2017). Hơn nữa, mẫu nghiên cứu tương đối trẻ tuổi, với hơn $60 \%$ đối tượng khảo sát trong độ tuổi 20 - 30. Một khi những người trẻ có cảm nhận thích thú, có trải nghiệm thú vị về công nghệ họ sẽ tái sử dụng dịch vụ và không màng lo lắng về quyết định của mình.

Yếu tố thói quen bị bác bỏ trong mô hình nghiên cứu này khi đánh giá tác động của thói quen đến sự tin tưởng và ý định tiếp tục sử dụng. Kết quả này tương đối khác biệt so với một số các kết quả nghiên cứu trước. Tuy nhiên, phù hợp với kết quả nghiên cứu của Jia, Hall, và Sun (2014) khi cho rằng NTD chưa có thói quen sử dụng ứng dụng di động để mua sắm khi dịch vụ này mới xuất hiện gần đây và nghiên cứu của Samira, Ofir, và Yufei (2017) khi cho rằng chưa đủ cơ sở kết luận thói quen tác động đến nhận thức rủi ro và sự tin tưởng khi mua sắm hàng hóa trực tuyến. Trên $50 \%$ số NTD trong mẫu khảo sát chỉ mới sử dụng ứng dụng đặt hàng thực phẩm thời gian gần đây và với tần suất sử dụng thấp từ 01 - 04 lần/tháng. Vì vậy, việc bác bỏ giả thuyết $\mathrm{H} 14$ và $\mathrm{H} 15$ có thể giải thích rằng ứng dụng đặt hàng thực phẩm mới xuất hiện gần đây tại Việt Nam, hầu hết NTD có rất ít kinh nghiệm và chưa thật sự hình thành thói quen sử dụng ứng dụng 
này. Kết quả này phù hợp với tình hình sử dụng ứng dụng di động ở mức độ thấp của người Việt Nam so với các nước láng giềng trong khu vực Đông Nam Á.

\section{Hàm ý quản trị}

Kết quả nghiên cứu đã cho thấy có nhiều yếu tố quan trọng tác động đến sự tin tưởng và ý định tiếp tục sử dụng ứng dụng này. Các nhà quản trị ứng dụng liên kết với nhà cung cấp thực phẩm có thể tiến hành xem xét và thực hiện việc:

\subsection{Tiếp tục thục hiện chuơng trình khuyến mãi và tăng tính giải trí cho úng dụng}

Thực tế nghiên cứu đã cho thấy giá và các chương trình khuyến mãi giúp NTD tin tưởng và tiếp tục sử dụng các dịch vụ giao hàng thực phẩm. Các nhà quản trị cần thực hiện nhiều chương trình khuyến mãi, mã giảm giá, miễn phí vận chuyển để cạnh tranh với các đối thủ trên thị trường. Đồng thời thiết kế ứng dụng sao cho vừa tạo được sự giải trí, kích thích khi tìm kiếm các chương trình khuyến mãi như lắc xu tích điểm hoặc sưu tầm vật phẩm để mang lại sự vui vẻ và thoải mái cho NTD. Đồng thời, kết quả khảo sát cũng cho thấy hành vi cài đặt nhiều ứng dụng giao hàng thực phẩm khác nhau trên cùng một thiết bị cũng cho thấy NTD dễ dàng từ bỏ một ứng dụng này để sử dụng ứng dụng khác. Do đó, nhà quản trị cũng cần kết hợp cải tiến các yếu tố khác để đảm bảo vẫn duy trì lợi nhuận hoạt động thay vì chỉ cạnh tranh về việc giảm giá và khuyến mãi.

\subsection{Nâng tầm ảnh hưởng xã hội đến thái độ và hành vi người tiêu dùng}

Nhà quản trị nên sử dụng hình thức quảng cáo với đại diện thương hiệu là những người nổi tiếng có tác động với nhóm đối tượng trẻ tuổi để gia tăng sự tin tưởng và ý định tiếp tục sử dụng đối với UDDD mới mẻ này. Ngoài ra, việc sử dụng các mã khuyến mãi hoặc tích lũy điểm thưởng khi NTD giới thiệu ứng dụng cho bạn bè/người thân là một cách thức mà các quản trị viên nên xem xét để nâng cao tính cạnh tranh cho ứng dụng của mình. Chất lượng thực phẩm, chất lượng giao hàng và chất lượng đường truyền tín hiệu của ứng dụng cũng làm nên lý do tích cực để lan truyền tin tốt lành về dịch vụ trong các thành viên gia đình, bạn bè và cộng đồng.

\subsection{Mang lại sụ̂ dễ dàng và tạo điều kiện thuận lọi khi NTD sử dụng úng dụng}

Đội ngũ thiết kế ứng dụng của doanh nghiệp cần thiết kế sao cho ứng dụng ngày càng đơn giản và thuận tiện, phù hợp với nhiều lứa tuổi và trình độ khác nhau. Giao diện ứng dụng cần trực quan đơn giản để giảm thiểu tối đa thời gian đặt hàng. Đồng thời, các nhà quản lý cũng cần có phương án dự phòng khi điện thoại không cài đặt được ứng dụng do không hỗ trợ hệ điều hành khi được nâng cấp và cập nhật ứng dụng mới khi có bất kì sự thay đổi. Ngoài ra, các kênh liên lạc như đường dây nóng, tổng đài điện thoại hoặc email phản hồi cần được quan tâm đúng mức để đảm bảo hỗ trợ cho khách hàng tốt nhất khi gặp sự cố.

\subsection{Nâng cao tính hiệu quả của úng dụng}

Để có thể cạnh tranh tốt trong việc tiết kiệm thời gian cho khách hàng, nhà quản trị cần mở rộng các cộng tác viên vận chuyển cũng như mở rộng hệ thống các cửa hàng thực phẩm trên ứng dụng để có thể đáp ứng kịp thời và nhanh chóng khi NTD có nhu cầu sử dụng. Việc này có thể thực hiện thông qua các hình thức như giảm chiết khấu cho các đối tác giao nhận để thu hút nhiều cộng tác viên vận chuyển hoặc giảm chiết khấu cho các cửa hàng khi kết nối và cung cấp thực phẩm cho ứng dụng. Ngoài ra, việc tích hợp công nghệ đặt hàng đảm bảo các cửa hàng thực phẩm có thể nhận ngay thông tin về các yêu cầu đặt món của NTD và chuẩn bị món ăn trong khi đối tác giao nhận đến lấy cũng có thể được tiết kiệm thời gian và nâng cao tính cạnh tranh cho ứng dụng của doanh nghiệp. 


\subsection{Nâng cao chất lựng thông tin của úng dụng}

Các ứng dụng nên trình bày thông tin và nội dung trên ứng dụng một cách rõ ràng, chi tiết và dễ hiểu để từ đó giúp khách hàng tin tưởng và tiếp tục sử dụng dịch vụ trong tương lai. Ngoài hình ảnh được miêu tả của món ăn thì các ứng dụng có thể miêu tả chi tiết về thành phần nguyên liệu hoặc cách chế biến của món ăn sẽ giúp NTD càng tin tưởng vào ứng dụng này. Nội dung hoặc hình ảnh các món ăn trên các cửa hàng cần được chuẩn hóa để tạo được sự hấp dẫn giúp các thực khách chọn lựa. Đồng thời, các nhà quản trị nên có đội ngũ chuyên trách để đánh giá và đảm bảo chất lượng thực phẩm và hình ảnh công bố trên ứng dụng phù hợp với thực tế.

\subsection{Nâng cao sụ̂ tin tưởng của NTD đối với úng dụng}

Việc nâng cao các yếu tố như kỳ vọng hiệu quả, kỳ vọng nỗ lực, ảnh hưởng xã hội, điều kiện thuận lợi, giá trị giá cả, chất lượng thông tin làm gia tăng sự tin tưởng hơn của NTD vào các ứng dụng và từ đó thúc đẩy ý định tiếp tục sử dụng các ứng dụng này trong tương lai. Sự tin tưởng này được thể hiện ở sự thực hiện đúng cam kết, ở đây có thể là thời gian giao hàng mà NTD cần phải đợi bao lâu, chất lượng thực phẩm có đúng với miêu tả trên ứng dụng hay không. Vì vậy, các quản trị viên cần đảm bảo đánh giá các cửa hàng thực phẩm về chất lượng cũng như thời gian giao hàng đã cam kết. Việc cung cấp chất lượng thực phẩm không đúng như hình ảnh không chỉ ảnh hưởng đến cửa hàng thực phẩm mà còn ảnh hưởng đối với các ứng dụng trong hiện tại và tương lai.

\section{Tài liệu tham khảo}

Ajzen, I. (1991). The theory of planned behavior. Organizational Behavior and Human Decision Processes, 50(2), 179-211.

Ajzen, I., \& Fishbein, M. (1975). Belief, attitude, intention, and behavior: An introduction to theory and research. San Francisco, CA: Addison-Wesley.

Akhlaq, A., \& Ahmed, E. (2013). The effect of motivation on trust in the acceptance of Internet banking in a low-income country. International Journal of Bank Marketing, 31(2), 115-125.

Alalwan, A. A. (2019). Mobile food ordering apps: An empirical study of the factors affecting customer e-satisfaction and continued intention to reuse. International Journal of Information Management, 50(2020), 28-44.

Appota. (2018). Vietnam mobile app market report. Retrieved April 10, 2020, from https://appota.com/uploads/report/Vietnam_mobile_app_market_Report_2018_EN.pdf

Brown, S. A., \& Venkatesh, V. (2005). Model of adoption of technology in the household, a baseline model test \& extension incorporating household life cycle. MIS Quarterly, 29(3), $399-426$.

Chai, S., \& Kim, M. (2010). What makes bloggers share knowledge? An investigation on the role of trust. International Journal of Information Management, 30(2010), 408-415.

Davis, F. D. (1989). Perceived usefulness, perceived ease of use, and user acceptance of information technology. MIS Quarterly, 13(3), 319-340.

E-Conomy SEA. (2019). E-Conomy SEA 2019 report. Retrieved April 12, 2020, from https://www.blog.google/documents/47/SEA_Internet_Economy_Report_2019.pdf

El-Masri, M., \& Tarhini, A. (2017). Factors affecting the adoption of e-learning systems in Qatar and USA: Extending the Unified Theory of Acceptance and Use of Technology 2 (UTAUT2). Educational Technology Research and Development, 65(3), 1-21. doi:10.1007/s11423-016-9508-8 
Escobar-Rodríguez, T., \& Carvajal-Trujillo, E. (2014). Online purchasing tickets for low cost carriers: An application of the Unified Theory of Acceptance and Use of Technology (UTAUT) model. Tourism Management, 43(2014), 70-88. doi:10.1016/j.tourman.2014.01.017

Flavian, C., Guinaliu, M., \& Gurrea, R. (2006). The role played by perceived usability, satisfaction and consumer trust on website loyalty. Information \& Management, 43(2006), $1-14$.

Fleming, J. H., \& Asplund, J. (2007). Human sigma: Managing the employee-customer encounter. Washington, DC: Gallup Press.

Garbarino, E., \& Lee, O. E. (2003). Dynamic pricing in internet retail: Effects on consumer trust. Psychology \& Marketing, 20(6), 495-513.

Gefen, D., Karahanna, E., \& Straub, D. W. (2003). Trust and TAM in online shopping: An integrated model. Computer Sciencepublished in Mis Quarterly 2003, 27(1), 51-90. doi:10.2307/30036519

Giovannini, C. J., Ferreira, J. B., Silva, J. F. D., \& Ferreira, D. B. (2015). The effects of trust transference, mobile attributes and enjoyment on mobile trust. Brazilian Administration Review, 12(2015), 88-108.

Hair, J. F., Black, W. C., Babin, B. J., \& Aderson R. E. (2014). Multivariate data analysis. Harlow, UK: Pearson Education Limited.

Hu, L., \& Bentler, P. (1999). Cut off criteria for fit indices in covariance structure analysis: Conventional criteria versus new alternative. Structural Equation Modeling, 6(1), 1-55.

Hwang, Y., \& Kim, D. J. (2007). Customer self-service systems: The effects of perceived web quality with service contents on enjoyment, anxiety, and e-trust. Decision Support Systems, 43(3), 746-760.

Indrawati, I., \& Putri, D. (2018). Analyzing factors influencing continuance intention of epayment adoption using modified UTAUT 2 model. International Conference On Information and Communication Technology, 6(2018), 167-173.

Jia, L., Hall, D., \& Sun, S. (2014). The effect of technology usage habits on consumers' intention to continue use mobile payments. Paper presented at the 20th Ameriacas Conference on Information Systems, Georgia, USA.

Kang, J. W., \& Namkung, Y. (2018). The information quality and source credibility matter in customer's evaluation toward food $\mathrm{O} 2 \mathrm{O}$ commerce. International Journal of Hospitality Management, 78(2018), 189-198.

Kuan, H. H., Bock, G. W., \& Vathanophas, V. (2008). Comparing the effects of website quality on customer initial purchase and continued purchase at e-commerce websites. Behaviour \& Information Technology, 27(1), 3-16.

Kustiwi, I. A., \& Isnalita, I. (2018). Trust and purchasing intention in e-commerce: Lazada Indonesia. Advances in Social Science Education and Humanities Research, 165(1), 28-32. doi:10.2991/iccsr-18.2018.7

Lee, S. W., Sung, H. J., \& Jeon, H. M. (2019). Determinants of continuous intention on food delivery apps: Extending UTAUT2 with information quality. Sustainability 2019, 11(11), Article 3141. doi:10.3390/su11113141 
Liao, C., Palvia, P., \& Lin, H. N. (2006). The roles of habit and web site quality in e-commerce. International Journal of Information Management, 26(6), 469-483.

Nicolaou, A. I., \& McKnight, D. H. (2006). Perceived information quality in data exchanges: Effects on risk, trust, and intention to use. Information Systems Research, 17(4), 332-351. doi:10.1287/isre.1060.0103

Panse, C., Rastogi, S., Sharma, A., \& Dorji, N. (2019). Understanding consumer behaviour towards utilization of online food delivery platforms. Journal of Theoretical and Applied Information Technology, 97(16), 4353-4365.

Rahi, S., Ghani, M. A., \& Muhamad, F. J. (2017). Inspecting the role of intention to trust and online purchase in developing countries. Journal of Socialomics, 6(1), 1-7. doi:10.41 72/2167-0358.1000191

Ranaweera, C., \& Prabhu, J. (2003). The influence of satisfaction, trust and switching barriers on customer retention in a continuous purchasing setting. International Journal of Service Industry Management, 14(4), 374-395.

Rosenberg, L., \& Czepiel, J. (1984). A marketing approach for customer retention. Journal of Consumer Marketing, 1(2), 45-51.

Samira, F., Ofir, T., \& Yufei, Y. (2017). A trust-risk perspective on social commerce use: An examination of the biasing role of habit. Internet Research, 27(3), 586-607.

Sharif, A., \& Raza, S. A. (2017). The influence of hedonic motivation, self-efficacy, trust and habit on adoption of internet banking: A case of developing country. International Journal of Electronic Customer Relationship Management, 11(1), 1-22.

Singh, A., Alryalat, M. A. A., Alzubi, J. A., \& Sarma, K. (2017). Understanding Jordanian consumers online purchase intentions: Integrating trust to the UTAUT2 framework. International Journal of Applied Engineering Research, 12(20), 10258-10268.

Statista. (2020). Online food delivery. Retrieved January 30, 2020, from https://www.statista.com/outlook/374/127/online-food-delivery/vietnam

Venkatesh, V., Morris, M., Davis, G., \& Davis, F. (2003). User acceptance of information technology: Toward a unified view. MIS Quarterly, 27(3), 425-478.

Venkatesh, V., Thong, J. Y. L., \& Xu, X. (2012). Consumer acceptance and use of information technology: Extending the unified theory of acceptance and use of technology. MIS Quarterly, 36(1), 157-178.

Verkijika, S. F. (2018). Factors influencing the adoption of mobile commerce applications in Cameroon. Telematics and Informatics, 35(6), 1665-1674.

We are Social. (2019). Digital 2020 Vietnam report. Retrieved April 15, 2020, from https://datareportal.com/reports/digital-2020-vietnam

Wijoseno, J., \& Ariyanti, M. (2017). Perceived factors influencing consumer trust and its impact on online purchase intention in Indonesia. International Journal of Science and Research, 6(8), 961-968. doi:10.21275/8081706 ISSN

Zhou, T. (2012). Examining mobile banking user adoption from the perspectives of trust and flow experience. Information Technology and Management, 13(1), 27-37. doi:10.1007/s 10799-011-0111-8 